\title{
Reflets
}

Revue ontaroise d'intervention sociale et communautaire

\section{Les centres de santé communautaire : la porte ouverte à la santé des francophones}

\author{
Centres de santé communautaire francophones de l'Estrie, Hamilton- \\ Wentworth-Niagam, Sudbury, Témiskaming, Toronto
}

Volume 5, numéro 2, automne 1999

La santé des francophones de l’Ontario

URI : https://id.erudit.org/iderudit/026275ar

DOI : https://doi.org/10.7202/026275ar

Aller au sommaire du numéro

Éditeur(s)

Reflets : Revue ontaroise d'intervention sociale et communautaire

ISSN

1203-4576 (imprimé)

1712-8498 (numérique)

Découvrir la revue

Citer cet article

Centres de santé communautaire francophones de l'Estrie, HamiltonWentworth-Niagam, Sudbury, Témiskaming, Toronto (1999). Les centres de santé communautaire : la porte ouverte à la santé des francophones. Reflets, 5(2), 162-175. https://doi.org/10.7202/026275ar

Tous droits réservés (C) Reflets : Revue ontaroise d'intervention sociale et communautaire, 1999
Ce document est protégé par la loi sur le droit d'auteur. L'utilisation des services d'Érudit (y compris la reproduction) est assujettie à sa politique d'utilisation que vous pouvez consulter en ligne.

https://apropos.erudit.org/fr/usagers/politique-dutilisation/ 


\section{Les centres de santé \\ communautaire : la porte ouverte à la santé des francophones}

D ocument préparé par les $C$ entres de santé communautaire francophones de l'E strie, H amilton-W entworth- $\mathrm{N}$ iagara, Sudbury, T émiskaming, Toronto.

La population francophone de l'O ntario a accès à cing $C$ entres de santé communautaire (C SC) francophones qui lui offrent des services de santé physique et mentale dans sa langue. Ces cinq centres évoluent dans des communautés différentes ayant des besoins différents, mais ils ont en commun l'utilisation de l'une des deux langues officielles de ce pays, le français. A insi, ils sont nés des efforts de la communauté francophone qui cherchait par ce moyen à améliorer l'accès aux services de santé. Ils sont situés à Cornwall, H amilton-Wentworth-N iagara, Sudbury, T émiskaming et Toronto.

$M$ algré les besoins spécifiques de chaque communauté, les cinq C SC francophones connaissent des réussites et des difficultés similaires qui les amènent aujourd'hui à présenter ce document. A près plusieurs années d'évolution, les CSC croient qu'il est maintenant temps de faire le point sur le rôle critique qu'ilsjouent dans le développement des communautés francophones qu'ils desservent. Les CSC francophones sont uniques et répondent souvent à une lacune du système de santé ontarien: les francophones n'ont pas accès à des soins de santé de qualité à cause de barrières linguistiques et culturelles. Les CSC ne dédoublent en aucun cas les services offerts à la communauté, mais complètent le travail des agences déjà en place. 
De plus, le niveau de santé des communautés francophones est encore inférieur à la population en général et les C SC francophones agissent non seulement au niveau de la santé des individus, mais également au niveau de la santé de la communauté en mettant sur pied, différents programmes basés sur les déterminants de la santé. $\mathrm{N}$ ous croyons que le rôle joué par les CSC francophones est essentiel. Le temps est donc venu de reconnaître cet apport en facilitant le fonctionnement de nos activités afin d'améliorer l'accès aux services de santé de base pour les francophones.

\section{Les CSC francophones : un mandat similaire aux autres Centres de santé communautaire, mais une réalité différente}

Les $C$ entres de santé communautaire sont des organismes communautaires à but non lucratif qui fournissent une gamme de services de santé primaires, des services en santé mentale, des services de nutrition, des programmes de promotion de la santé et de développement communautaire dans plus de 55 communautés en 0 ntario. Ilssont toujoursorientés verslesusagers. Tousles C SC ont le même mode de fonctionnement: un mandat unique, une population unique et des programmes et des services adaptés aux besoins de leur communauté. Ils sont gérés par des conseils d'administration qui représentent la communauté desservie et qui agissent comme transmetteurs des besoins véritables à combler. Par le biais d'une équipe multidisciplinaire, les CSC sont en mesure de fournir des soins continus et des services de grande qualité aux clients éprouvant des besoins en santé. Leur personnel est salarié et peut inclure des médecins, des infirmiers, des travailleurs communautaires, des promoteurs de la santé, des diététistes et des travailleurs en santé mentale ainsi qu'une coordonnatrice du développement communautaire. 
II existe maintenant cinq CSC qui offrent des services de santé communautaire à la population francophone spécifiquement. Le doyen des centres, le Centre médico-social communautaire de Toronto, a vu le jour en 1988 et le benjamin, le Centre de santé communautaire du T émiskaming, a ouvert ses portes en 1995. Les trois autres C SC desservent les communautés francophones de Cornwall, $\mathrm{H}$ amilton-Wentworth- $\mathrm{N}$ iagara et Sudbury.

Le mandat donné aux C SC francophones est similaire à celui des autres $C$ entres de santé, mais les services qu'ils offrent s'adressent uniquement aux personnes s'exprimant en français plutôt que par secteur géographique ou encore, par type de clientèle (e.g. réfugiés, sidatiques, etc.). La gamme de services mis sur pied par les C SC francophones est donc très vaste. Elle varie aussi d'un C SC à l'autre. En comparaison, les services des C SC anglophones sont destinés à une clientèle beaucoup plus pointue et peuvent s'appuyer sur d'autres ressources dansleur communauté.

La clientèle des CSC francophones n'est pas homogène, les seuls liens les unissant étant la langue et la culture. Le menu des services des C SC francophones doit donc s'adapter à toutes les sauces et faire preuve d'une très grande flexibilité. Les professionnels jonglent avec toutes les facettes de la santé des individus et n'ont souvent pas le luxe de concentrer toute leur énergie à s'attaquer à une problématique particulière.

U ne différence importante entre les CSC anglophones et francophones demeure le statut dont ils jouissent dans leur communauté. Si les C SC anglophones peuvent être vus comme une alternative aux autres organismes de soins de santé, les C SC francophones sont la porte d'entrée privilégiée du système de santé ontarien pour les francophones qui désirent obtenir des services dans leur langue. $U$ ne autre différence fondamentale est que les C SC francophones doivent adapter leurs services pour tenir compte des facteurs culturels de la population francophone de I'O ntario, au même titre que les C SC qui s'adressent à une communauté chinoise ou autochtone.

A ujourd'hui encore, malgré l'adoption de la $L$ oi sur les services en français de 1986, les organismes de services de santé offrent peu, pour ne pas dire pas du tout, de services structurés en français 
et ce, même dans les régions désignées par la $L$ oi. $D$ ans un tel contexte, le C entre de santé communautaire devient l'organisme de référence pour les francophones préoccupés par leur santé. O n y vient pour consulter le médecin de famille, pour être référé à un spécialiste ou encore pour obtenir des services d'interprétation. Les francophones s'adressent aussi à leur C entre de santé pour obtenir de la documentation en français sur un sujet qui touche la santé ou pour obtenir de l'aide lorsqu'ils ont le mal de vivre. Le Centre de santé communautaire devient un lieu rassembleur où les francophones sont assurés d'être compris. Par le fait même, les C SC francophones subissent beaucoup de pressions de la part de leur clientèle. La communauté ne pouvant se tourner vers d'autres sources, les CSC se voient obliger d'en faire plus avec souvent moins de moyens que les agences de santé mandatées pour offrir les services de base.

\section{L'impact des CSC francophones dans leur communauté}

Les cinq C SC francophones ont eu un impact certain dans leur communauté. L'histoire à succès se répète d'une ouverture d'un CSC à l'autre: la clientèle afflue facilement, les services offerts ne semblent jamais suffisant. C es centres de santé multidisciplinaires ne font pas que répondre à une pénurie de services en français, ils intègrent la culture des communautés francophones desservies, ce qui est essentiel pour les amener à se prendre en main. Les centres accumulent souvent lescas lourds et compliqués qui étaient pauvrement ou pas du tout desservis auparavant.

En effet, laformule des C SC est idéale pour toute la population, et pour les francophones en particulier. Les références à des spécialistes sont faites au besoin et on y offre des servicesà peu de frais, car les professionnels sont des salariés. Ilstraitent plusque les symptômes des problèmes de santé des individus. Ils cherchent aussi à en éradiquer la source par des programmes d'éducation et par des éléments incitatifs pour changer les habitudes de vie. 


\section{Une réponse favorable au Témiskaming}

Lesfrancophones font souvent face à des entraves des plus subtiles. A u T émiskaming, entres autres, aucun organisme de santé a un plan de désignation en vertu de la $L$ oi 8 , pour offrir des services en français. Toutefois, des services en français sont offerts, mais uniquement si la demande est suffisante. $0 \mathrm{r}$, les francophones ne demandent pas un service qui n'existe pas. Lorsque le temps fut venu de discuter de la mise sur pied d'un C SC francophone au T émiskaming, les opposants avancèrent l'idée qu'il n'y avait pas de besoin pour un tel centre, car il n'y avait pas de demande pour des services en français. L'histoire se chargea de leur prouver le contraire!

D epuis l'ouverture du C SCT en décembre 1995, la réponse de la population francophone a été plus que favorable. En un peu plus d'un an, on y dénombre plus de 1000 dossiers, plus de 4200 visiteset plus de 4000 personnes danslesactivités communautaires. Les conférences communautaires attirent régulièrement une cinquantaine de personnes, les sall les d'attente sont pleines à craquer et les périodes d'attente s'allongent sans cesse. Le triomphe du CSCT est la preuve que les besoins en matière de santé ne sont rencontrésque lorsque des services formels et structurés sont mis en place. En somme, le succès du C SCT repose sur une stimulation constante de la demande par des stratégies de marketing adaptées à la population francophone et desprogrammesqui correspondent à leurs besoins.

D ans le domaine de la santé, la façon de communiquer avec le client est très importante: les messages transmis doivent être clairement énoncés et le client doit être en mesure de les comprendre. Le bilinguisme est souvent d'usage courant chez les francophones, ce qui leur permet d'être fonctionnels dans un milieu anglophone; sans toutefois avoir une maitrise totale de la langue anglaise. Ainsi, quand il devient nécessaire pour eux d'exprimer en détail leurs malaises, le vocabulaire anglophone leur fait souvent défaut. $\mathrm{N}$ otons que les personnes âgées francophones sont particulièrement vulnérables, car une grande proportion d'entre eux est unilingue. 
U ne communication efficace en langue française entraîne de meilleurs soins: les risques d'erreurs dans l'interprétation du diagnostic sont réduits (le professionnel étant en mesure de saisir toutes les nuances des malaises énoncés et de demander des éclaircissements), le service est effectué plus rapidement et il y a moins de visites médicales, puisque le client a bien compris le message et le traitement. La personne francophone garde ainsi son capital santé plus longtemps ce qui s'avère rentable pour la société.

\section{Une histoire à succès du Centre de santé communautaire de Sudbury}

Dans la région de Sudbury, le pourcentage de personnes qui fument est très élevé. C ette tendance est encore pire chez les francophones. Bien que plusieurs organismes locaux offrent des sessions de groupe pour arrêter de fumer, aucun n'a jamais réussi à rejoindre un nombre suffisant de francophones pour organiser des sessions en français. Dès que le C SC S a annoncé qu'il s'apprêtait à offrir des sessions pour arrêter de fumer, la demande a été très grande. II y a eu suffisamment de participants et participantes pour former deux groupes d'un peu plus de 20 personnes dans la ville de Sudbury et un autre de quatorze personnes à $\mathrm{R}$ ayside- $\mathrm{B}$ alfour. $\mathrm{C}$ et exemple se répète dans presque tous les programmes du C SC S. Lorsque les services en français sont vraiment disponibles, les francophones les utilisent.

En plus d'aider les francophones à se prendre en main sur le plan individuel, lesC SC jouent un rôle critique dans la dynamique entre les autres organismes de leur communauté. C haque C SC francophone agit comme un élément catalyseur qui permet d'entreprendre un dialogue et de développer des partenariatsentre les organismes déjà en place, anglophones ou bilingues, mais qui s'activaient auparavant en parallèle.

Plusieurs organismes devant offrir des services en français et ayant la volonté de le faire réu ussissaient mal à rejoindre la clientèle francophone. L'arrivée d'un C SC qui s'affiche en français leur a ouvert la porte. Le C SC devient alors un interlocuteur avec qui 
ilspeuvent travailler afin de développer des services adéquats pour lesfrancophones. L'effet mobilisateur que provoque les C SC dans les communautés francophones permet de créer un réseautage entre organismes qui élimine la duplication de services et favorise la prise en charge du milieu par la communauté.

La présence d'un CSC francophone a aussi un effet de valorisation pour la communauté francophone qui y voit le début d'une reconnaissance de ses besoins. Les francophones ont trop souvent été traités comme des citoyens de deuxième classe par un système de santé peu préoccupé de lui donner des services dans leur langue. L'accès à un centre de santé où les services sont dispensés avec rigueur et professionnalisme redore le blason d'une communauté laissée à elle-même. À juste titre, il serait souhaitable qu'un Centre de santé communautaire francophone soit érigé dans toutes les régions où vit une communauté francophone.

\section{Les réalités particulières aux CSC francophones}

\subsection{Les avantages reliés aux CSC francophones}

Les cinq CSC francophones ontariens rencontrent bien des embûches et des difficultés sur leur parcours afin d'œuvrer auprès de leurs communautés. D es frais supplémentaires sont imputables à ces difficultés, frais que les autres C SC n'ont pas. Les CSC francophones s'avèrent être l'outil par excellence pour rejoindre les communautés francophones que le système traditionnel de santé ne parvenait pas à desservir. Le prix à payer est peu élevé comparativement au coût social engendré par le déclin de la santé d'un segment de la population.

Les C SC visent à déterminer les besoins de la population et à y répondre adéquatement en offrant une gamme de services de santé intégrés. Les C SC sont des centres multiservices de santé adaptés aux besoins de la population francophone. Ils ont un accès simplifié, en étant un guichet unique à l'ensemble des professionnels de la santé. Ils permettent d'effectuer des dépistages 
précoces et des interventions ciblées vers les personnes en besoin afin d'éviter que leur situation ne se détériore. Enfin, en atteignant des populations difficiles à rejoindre par le système de santé traditionnel (hôpitaux, bureaux de santé publique, cliniques médicales, etc.), ils sont une valeur ajoutée au système de santé et ils réduisent ainsi les coûts rattachés à la mauvaise utilisation des services des autres organismes.

\section{Cornwall : spécificité des Centres de santé communautaire}

Au C entre de santé communautaire de l'Estrie (CSCE), comme dans plusieurs autres C SC, une gamme diversifiée de services est offerte. C es services sont en concordance avec la philosophie des CSC, c'est-à-dire de développer la solidarité et la responsabilité de la clientèle. De plus, les études démontrent que les C SC sont moins coûteux au système de santé.

À titre d'exemple, les professionnels des C SC francophones (médecin, infirmière, travailleur social) se déplacent au domicile des clients. C es besoins de services sont souvent dus au manque de services en français dans les hôpitaux ou les centres pour personnes âgées. A insi, les personnes souffrantes préfèrent souvent demeurer au foyer, entourées des leurs pour vivre leurs derniers moments, particulièrement celles en phase terminale de cancer. Elles s'y sentent mieux comprises. II leur faut donc un support médical, infirmier et social. C'est également le cas des personnes handicapées ou des personnes âgées ne pouvant se déplacer.

Chaque visite du médecin, accompagné d'une infirmière, coûte environ 200 \$. Si le client était hospitalisé, il en coûterait 700 à 800 \$ par jour. Le client qui demeure à domicile reçoit une ou deux visites du médecin par semaine. Imaginez l'économie! De plus, cette façon de faire responsabilise les membres de la communauté tout en leur donnant le soutien nécessaire pour prendre en main leur santé et celle de leurs proches. Bref, il s'agit là d'un exemple d'efficacité et d'efficience.

Leur force première réside dans leur grande capacité d'adaptation et la souplesse de leur structure qui leur permettent 
de répondre rapidement et à faible coût à des situations problématiques soulevées par la communauté qu'ils desservent. Les C SC favorisent la participation desmembres de la collectivité à des activités qui visent la promotion de la santé, la prévention de la maladie, la promotion d'habitudes de vie saine et I'intervention précoce. Ils offrent un milieu propice au soutien par les membres de la collectivité et à l'épanouissement de leur communauté.

De plus, on peut observer que dès qu'un C SC s'implante dans une région, l'offre de services en français des organismes déjà en place s'accroît. Les C SC semblent avoir un effet d'entraînement, ce qui rend l'ensemble de la communauté francophone doublement gagnante. $U$ ne personne qui a besoin d'un service de santé n'est pas dans une position qui lui permet de l'exiger. Le C SC francophone n'est pasle malade et peut exiger le service pour ses clients.

\section{Une augmentation des interventions au CMSC de Toronto}

Le $C$ entre médico-social communautaire deToronto a ouvert ses portesil y a maintenant onze ans. II dessert une clientèle étendue sur tout le territoire du Toronto métropolitain avec deux points de services, I'un au centre ville deToronto et l'autre situé à $\mathrm{N}$ orth York.

C es deux dernières années, le CM SC a multiplié les efforts pour consolider sa présence dans la communauté francophone. G râce au développement de partenariats avec d'autres organismes, anglophones et francophones, à la production d'un bulletin d'information expédié un peu partout dans la communauté et, surtout, à une campagne de promotion bien orchestrée, le C M SC a presque doublé son volume d'activités. Le CM SC a dénombré 5500 interventions pour l'année 1993-1994. En 1996-1997, le CM SC a procédé à 9120 interventions!

Le CM SC est un organisme imprégné de la même culture et de la même langue que lesclientsqu'il vise. C ela n'est pas étranger à son succès. La connaissance du milieu qu'il possède lui a permis 
de viser juste pour rejoindre sa clientèle et en arriver à une augmentation de I'utilisation de ses services. De plus, en s'engageant dans la vie communautaire de la francophonie torontoise, le CM SC aide les francophones, clients ou non, à prendre leur santé en main par des programmes en prévention et en promotion de la santé.

\subsection{Les difficultés et les embûches dans la prestation des services}

Les C SC francophones font face au problème de l'éparpillement de leur clientèle. Par exemple, les francophones sont dispersés dans plusieurs quartiers de la ville de Toronto, de Sudbury ou encore, dans plusieurs municipalités de Cornwall, $\mathrm{H}$ amiltonWentworth-N iagara ou Témiskaming. C ette situation a un impact certain sur les budgets qui leur sont accordés et sur les stratégies utilisées pour rejoindre leur clientèle, si on les compare à un C SC desservant une population géographiquement délimitée comme Sandy $\mathrm{H}$ ill à $\mathrm{O}$ ttawa.

$R$ ejoindre la clientèle est un défi à affronter sous deux angles: les services et la promotion. M ême si la plupart des C SC francophones offrent leurs services de santé primairesà des points centralisés, certains programmes de promotion de la santé et services à domicile demandent des déplacements de la part du personnel des centres. Les frais de transport et de location de salles s'accumulent donc très rapidement. La promotion des services peut devenir dispendieuse lorsque l'on doit acheter des espaces publicitaires dans plusieurs médias. Lesfraissupplémentai res que tout cela engendre ne sont pas reflétés dans les budgets types accordés aux C SC francophones par les bailleurs de fonds.

\section{Les succès du partenariat avec les organismes anglophones}

Les dirigeants du $\mathrm{C}$ entre de santé communautaire de $\mathrm{H}$ amilton et $\mathrm{N}$ iagara ont compris l'importance de développer desliensavec les agences anglophones et ilsy ont consacré beaucoup d'efforts depuis trois ans. Les partenariats qu'ils ont établis avec les agences 
anglophones élargissent la gamme de services disponibles et font mieux connaître le $C$ entre dans la communauté. En général, ces agences collaborent aisément avec le C entre d'autant plusqu'elles sont souvent incapables de desservir des clients francophones qui parfois ne parlent aucun mot d'anglais, cela en dépit du mythe qui veut que «tous les francophones parlent anglais ».

Voici quelques exemples de partenariats développés par le Centre de santé communautaire de $\mathrm{H}$ amilton et $\mathrm{N}$ iagara qui profitent aux communautés francophones qu'il dessert.

Le partenariat avec l'abri $\mathrm{M}$ artha $\mathrm{H}$ ouse de $\mathrm{H}$ amilton permet aux familles francophones à risque d'avoir accès gratuitement à des camps d'hiver et d'été extraordinaires, où parents et enfants participent aux activités type counselling et aux autres activités de détente. D e même, la collaboration avec l'abri $M$ artha H ouse et Inasmuch House assure un abri temporaire aux femmes francophones et leurs enfants sans abri et aux réfugiées francophones. Le C entre s'assure que ces familles francophones sont servies en français grâce aux services d'interprétation qu'il offre gracieusement aux agences avec lesquelles il collabore.

Avec le Sexual Assault Centre de $\mathrm{H}$ amilton (SACHA), le $\mathrm{C}$ entre de santé communautaire de $\mathrm{H}$ amilton et $\mathrm{N}$ iagara a développé un partenariat axé sur la formation d'intervenantes pour la ligne téléphonique de crise destinée aux femmes francophones. C e partenariat a aussi fait en sorte que les femmes francophones qui appellent sur la ligne de crise anglophone peuvent être servies en français, si elles le désirent.

U $n$ autre problème endémique semble être le manque de matériel disponible en français. Q ue l'on parle des brochures, du matériel didactique pour les animateurs d'ateliers, de dépliants d'éducation en matière de santé, de logiciel d'ordinateur, tout est difficile ou quasi impossible à obtenir en langue française. Lorsque les documents gouvernementaux conçus pour la clientèle arrivent enfin en version française, bien longtemps après l'apparition du document original, ils sont souvent périmés ou dépassés. Les professionnels des CSC francophones dépensent beaucoup de temps et d'énergie à trouver du matériel de qualité en français ou à traduire eux-mêmes les outils d'information essentiels à leur 
travail d'éducation auprès de leur clientèle. U tiliser du matériel provenant du Q uébec, par exemple, demande aussi beaucoup de temps d'adaptation au niveau de la terminologie et des références.

Le recrutement et la formation d'un personnel qualifié sont une barrière bien difficile à franchir pour les C SC francophones. La qualité de la communication avec le client est un des éléments les plusimportants pour lui assurer un bon service dansle domaine de la santé physique et mentale. Les professionnels embauchés doivent être compétents et être en mesure des'exprimer en français couramment et en anglais afin de s'intégrer au restant du système de santé local. Lesfrancophones sont presque absents dans certaines professions médicales en 0 ntario et les personnes bilingues sont rares.

Les C SC francophones doivent multiplier les démarches pour combler les postes qu'ils ont à offrir, surtout pour le recrutement de médecinset, danscertains cas, de travailleurs sociaux. B eaucoup de frais sont engagés pour afficher les postes un peu partout dans la province, et au-delà de ses frontières, en offrant des salaires qui ne sont souvent pas compétitifs compte tenu du manque de ressources.

Par exemple, le ministère de la Santé ne considère pas la région de Toronto comme une région sous-desservie en effectif de médecins. La réalité francophone est tout autre: le CSC francophone de Toronto ne parvient pas à trouver un médecin francophone ou bilingue sur le territoire de la région. II doit s'adresser à l'extérieur. Le C SC n'a pourtant pasl'avantage d'offrir un salaire plus élevé pour inciter un médecin de l'extérieur à s'installer chez lui puisqu'il est dansune zone sur- desservie pour les institutions anglophones. La période de recrutement est très longue et très ardue sans les outils incitatifs nécessaires. À Sudbury, le problème se répète à une différence près. La ville de Sudbury est reconnue sous- desservie par le ministère de la Santé, mais la branche qui finance les médecins ne le reconnaît pas.

U ne fois le personnel en place, les C SC francophones voient surgir une autre difficulté: le domaine de la santé est en constante évolution et les professionnels qui s'y activent doivent mettre à jour leurs connaissances régulièrement. Lesoccasions de formation 
et de perfectionnement en français sont une denrée rare. Les professionnels qui ne se sentent pas à l'aise pour suivre une formation en anglais sont obligés de se joindre à des ateliers ou à des groupes de formation souvent bien éloignés, que ce soit à I'université Laurentienne de Sudbury ou à M ontréal. Les frais encourus par les C SC sont d'autant plus élevés.

Les dirigeants des C SC sont appelés à siéger à toutes sortes de comités gouvernementaux, leurs expertises et leurs connaissances du milieu ne pouvant être mise en doute. Bien qu'en soi, ce travail convient au rôle des CSC, il exige l'investissement de beaucoup de temps et d'argent, deux facteurs qui leur manquent abondamment. La présence des C SC francophones à ces comités est importante puisqu'ils doivent être aux premiers rangs pour s'assurer que l'on tient compte des francophones lors de la mise en place de nouveaux services ou lors du réseautage des services existants sur le plan régional et provincial.

É voluer en langue française dansun contexte minoritaire n'est pas chose facile pour un organisme qui rencontre un échéancier serré. Par exemple, les délais causés par la recherche d'une firme d'expert conseil pour produire des rapports en français peuvent ternir l'image de rigueur de l'organisme en question qui ne cherche qu'à obtenir un service de qualité. Et pourquoi ne pas parler des coûts supplémentaires encourus pour installer une imprimante avec un mode d'emploi «Do it yourself ». II faut payer un programmeur pour arriver à la faire fonctionner... avec des logiciels en français.

En somme les réalités particulières aux C SC francophones sont parfois difficiles à comprendre. II en résulte que leurs professionnels consacrent une somme appréciable de leur temps à justifier leur existence et à clarifier certaines situations problématiques avec les autres populations. Toutefois, ils facilitent I'accès à des soins de santé pour ces derniers, car ils s'accaparent une partie de la population, ce qui libère l'accès aux autres services de santé. 


\section{Une reconnaissance officielle de l'apport des CSC francophones et de ses besoins}

Les C SC francophones jouent un rôle de premier plan auprès des communautés francophones où leur arrivée a provoqué un impact chez les individus et chez les organismes, anglophones et francophones, du milieu. Lesinstances gouvernementales doivent se réjouir de l'intégration accomplie en quelquesannées seulement par les C SC, car ils contribuent à peu de frais à conserver une partie de la population en meilleure santé.

Comme ils répondent à une pénurie de services et font face à une clientèle dispersée, les C SC francophones sont débordés de demandes de services qu'ils arrivent de peine et de misère à combler. Leurs demandes de budget aux bailleurs de fonds doivent être effectuées à la pièce et sont évaluées sur le même pied que celles de d'autres organismes qui peuvent compter sur plus de ressources dans leur communauté environnante.

Les C SC francophones demandent au ministère de la Santé de I'O ntario de reconnaître l'apport particulier qu'ils font à leur communauté en tenant compte du contexte spécifique où ils évoluent. En terme concret, le ministère doit reconnaître que les budgets de fonctionnement des CSC francophones doivent être révisés et qu'une politique particulière doit être mise en place pour évaluer leurs besoins en fonction de la spécificité de leur population, des services de base exigés et des distancesà parcourir. Demander que les services médicaux soient disponibles 24 heures par jour, sept jours par semaine avec 1,5 ETP (équivalent temps plein) de médecin n'est pas raisonnable. Le ministère doit aussi reconnaitre que les CSC offrent des services essentiels à la population francophone et il doit les aider à conserver leur acquis, qui ne sont que primaires, et à développer de nouveaux services.

Ces acquis de services en santé pour les francophones sont fragiles et risquent de s'effondrer s'ils sont soumis aux impératifs régionaux. Le ministère de la Santé, pour assurer un traitement équitable de la population francophone ontarienne, doit protéger 
les services existants par une politique provinciale qui s'engage à les desservir dans le respect de leur langue et de leur culture et ce, au même titre que les anglophones.

Les C SC francophones demandent aussi au ministère de tenir compte de la place de choix que les CSC ont pris dans les communautés francophonesavant de mettre sur pied de nouveaux services de soins primaires dans une région. A près quelques années d'exercice, les C SC sont perçus, par lesindividuset lesorganisations de leur communauté, comme la source de références pour tout ce qui touche la santé des francophones. Le ministère peut améliorer l'accès aux services de santé pour les francophones en reconnaissant le rôle de pivot joué concrètement par les C SC dans leur communauté. Cette reconnaissance devrait se traduire par le choix desC SC comme maître d'œuvre pour la communauté francophone, de l'établissement des services découlant des grandes réformes en santé des gouvernements telles celles des soins de longue durée et de la réforme de la santé mentale.

En terminant, le ministère doit reconnaître que les C SC sont souvent les seuls fournisseurs de services structurés en français et qu'ils sont aux premiers rangs pour évaluer et répondre aux besoins de leur communauté. Leurs demandes d'ajouts de services spécialisés (ergothérapie, travailleurs sociaux, etc.) ne visent pas à augmenter la clientèle de leur centre ou à récupérer des services existants dans un autre organisme, mais bien à offrir un service de base. 